\title{
Implicit Finite Difference Scheme for Singularly Perturbed Burger-Huxley Equations
}

\author{
KABETO Masho Jima*and DURESSA Gemechis File \\ Department of Mathematics, College of Natural Sciences, Jimma University, Jimma, \\ Ethiopia.
}

Received 5 April 2021; Accepted 11 June 2021

\begin{abstract}
In this paper, an implicit finite difference scheme is presented to solve one dimensional unsteady singularly perturbed Burger-Huxley equation. The quadratically convergent quasilinearization technique is used to linearize the nonlinear term of the equation. The innovative significance of this paper is the procedure to consider initial guesses in order to start the quasilinearization technique. This basic initial guessing causes to produce a more accurate solutions with the small iteration number for the problem under consideration. The derivatives are replaced by finite difference approximation, then we obtain the two-level time direction and the three-term recurrence relation in the spatial direction. The convergence analysis of the proposed method has been established. Numerical experiments were conducted to support the theoretical results. Further, the result shows that the proposed method gives a more accurate solution with a higher rate of convergence than some existing methods.
\end{abstract}

AMS Subject Classifications: 65M06, 65M12, 65M15

Chinese Library Classifications: O175.29

Key Words: Singularly perturbed; Burger-Huxley equation; higher-order; accurate solution.

\section{Introduction}

Consider one-dimensional unsteady singularly perturbed Burger-Huxley equation of the form:

*Corresponding author. Email addresses: maashookoo .reemii@gmail . com (M. J. Kabeto), gammeef@gmail . com (G. F. Duressa) 


$$
\begin{cases}\mathcal{L}_{x, \varepsilon} u(x, t) \cong-\varepsilon \frac{\partial^{2} u}{\partial x^{2}}+\alpha u \frac{\partial u}{\partial x}+\frac{\partial u}{\partial t}-\beta(1-u)(u-\gamma) u=0, & \forall(x, t) \in \mathcal{D}, \\ u(x, 0)=u_{0}(x), & x \in \bar{\Omega}=[0,1], \\ u(0, t)=s_{0}(t), & t \in(0, T], \\ u(1, t)=s_{1}(t), & t \in(0, T],\end{cases}
$$

where $0<\varepsilon \ll 1$ is a perturbation parameter. The solution domain $\mathcal{D}=(0,1) \times(0, T]$, and $\alpha \geq 1, \beta \geq 0, \gamma \in(0,1)$ are given constants. Such equation describes the interaction between convection, diffusion and reaction processes [1]. Burger-Huxley equation describes numerous fascinating phenomena such as busting oscillation [2], interspike [3], population genetics [4], bifurcation and chaos [5]. In the former few decades, several analytical methods were suggested to solve the Burger-Huxley equation. For example, by using Hirota method, Satsuma [6] obtained an exact solitary solution for this equation. Wang and other researchers in [7] built an exact solitary wave solution of the generalized Burger-Huxley equation. In [8], Wazwaz constructed some travelling wave solutions for the generalized forms of Burgers, Burgers-KdV and Burger-Huxley equation by using the standard tanh-function technique. The most innovative and robust numerical methods have been conducted on several families of singularly perturbed parabolic problems like in, [9-13].

Recently, several researches give attention to solve Burger-Huxley equation by the numerical methods such as different classes of domian decomposition method [14-17], variational iteration method [18], finite difference scheme [19,20], different kinds of spectral methods [21,22], family of collocation method [23,24], computational meshless method [25]. However, the nature of the solution of singularly perturbed Burger-Huxley problem exhibits boundary layer. Because of the occurrence of this layer, the above mentioned methods are in question and known to be insufficient to estimate the accurate solution.

Consequently, to obtain uniformly convergent method, Kaushik and Sharma [26] establishes on nonuniform mesh for solving Eq. (1.1). Gupta and Kadalbajoo [27] assembled a numerical scheme that contains of implicit-Euler method which is first order uniformly accurate to discretize in temporal direction on a uniform mesh and a monotone hybrid finite difference operator to discretize the spatial direction with a piecewise uniform Shishkin mesh.

Research has been conducted to improve on the performance of Shishkin meshes while recalling some of their simplicity. The use of a piecewise uniform mesh with choosing transition parameter. The extendibility of the methods using meshes of Shishkin type to higher dimensional problem clarifies why people are interested in using them. Another advantage of Shishkin meshes over other types of meshes, is the convenience to handle complicated higher-order methods. Thus, in this paper, we aim at formulating higherorder fitted mesh of Shishkin type to solve the singularly perturbed Burger-Huxley equations. 
Evidently, the methods obtainable in $[26,27]$ are of the layer-adapted mesh approach. Moreover, recently Liu, Li-Bin, and others in [1] constructed a robust adaptive grid method for the problem (1.1). However, the obtained accuracy of the solution needs improvement which indicates that still for such kind of problems requests to develop another numerical method to produce a more accurate numerical solution. To serve this purpose, we will devise more accurate, stable, and consistent scheme for the problem under consideration. We first linearized the nonlinear terms by using quasi-lineariztion technique. Then, the average finite difference approximation is applied to approximate the derivatives of the differential equation. The convergence analysis is given to show that the presented method is second-order convergent.

\section{Properties of Continuous Solution}

In this section, a priori estimate for the solution of Eq. (1.1) on the solution domain denoted by $\mathcal{D}$. Suppose the base boundary $\Gamma_{b}=\{(x, t): t=0, x \in[0,1]\}$, left boundary $\Gamma_{l}=$ $\{(x, t): x=0, t \in[0, T]\}$ and right boundary $\Gamma_{r}=\{(x, t): x=1, t \in[0, T]\}$, then $\partial \mathcal{D}=\Gamma_{l} \cup \Gamma_{b} \cup \Gamma_{r}$. For any given function $g(x, t) \in C^{0} \overline{\mathcal{D}}$, the maximum norm is defined as follows

$$
\|g(x, t)\|_{\overline{\mathcal{D}}}=\max _{(x, t) \in \overline{\mathcal{D}}}|g(x, t)| .
$$

Lemma 2.1. (Maximum Principle): Let $v(x, t) \in C^{2,1}(\overline{\mathcal{D}})$, be a smooth function such that $\mathcal{L}_{x, \varepsilon} v(x, t) \geq 0$, where $\forall(x, t) \in \mathcal{D}$ and $v(x, t) \geq 0$ on $\partial \mathcal{D}$. Then, $v(x, t) \geq 0, \forall(x, t) \in \overline{\mathcal{D}}$.

Proof. The proof of this Lemma is done on [27].

A direct importance of this maximum principle is to establish the following stability estimte.

Lemma 2.2. Let $u(x, t)$ be the solution of problem (1.1), then we have,

$$
\|u\|_{\overline{\mathcal{D}}} \leq T\left\|u_{0}\right\|_{\Gamma_{b}}+\|u\|_{\partial \mathcal{D}}
$$

To drive the convergence and stability of the time direction, we give the bounds on the time derivatives as follows:

Lemma 2.3. Let $u(x, t)$ be the solution of problem (1.1), then there exists a constant $C$, independent of $\varepsilon$, such that

$$
\left|\frac{\partial^{i} u(x, t)}{\partial t^{i}}\right| \leq C, \quad i=0,1,2, \forall(x, t) \in[0,1] \times(0, T] .
$$

Proof. It follows from lemma (2.2), we can obtain $|u(x, t)| \leq C$. Next one can obtain the first-order derivative bound with respect to time variable as follows. Differentiating the 
initial funtion concerning to the space vatiable as $\partial u(x, 0) / \partial x=u_{0}^{\prime}(x)$ and $\partial^{2} u(x, 0) / \partial x^{2}=$ $u_{0}^{\prime \prime}(x)$, which yields

$$
\varepsilon \frac{\partial^{2} u(x, 0)}{\partial x^{2}}-\alpha u(x, 0) \frac{\partial u(x, 0)}{\partial x}+\beta(1-u(x, 0))(u(x, 0)-\gamma) u(x, 0)=0, \quad x \in[0,1] .
$$

On the boundaries $x=0$ and $x=1$, we obtain $\partial u(x, 0) / \partial t=s_{0}^{\prime}(t), \partial u(1, t) / \partial t=s_{1}^{\prime}(t), t \in(0, T]$. Therefore, for sufficiently smooth functions $u_{0}(x), S_{0}(t)$ and $S_{1}(t)$, there exists a constant $C_{1}$, such that

$$
\left|\frac{\partial^{i} u(x, t)}{\partial t^{i}}\right| \leq C_{1}, \quad \forall(x, t) \in \partial \mathcal{D} .
$$

For $\forall(x, t) \in(0,1) \times(0, T]$, it follows from Eq. (1.1) that

$$
\mathcal{L}_{x, \varepsilon} \frac{\partial u(x, t)}{\partial t}=0
$$

As the operator $\mathcal{L}_{x, \varepsilon}$ is uniformly stable, using lemma (2.2), we get

$$
\left|\frac{\partial u(x, t)}{\partial t}\right| \leq C_{1}, \quad \forall(x, t) \in \partial \overline{\mathcal{D}} .
$$

Similarly, we get the bound for the second-order derivative $\partial^{2} u(x, t) / \partial t^{2}$.

\section{Formulation of the Numerical method}

Consider Eq. (1.1), which re-written as

$$
\begin{cases}\frac{\partial u}{\partial t}-\varepsilon \frac{\partial^{2} u}{\partial x^{2}}=F\left(x, t, u, \frac{\partial u}{\partial x}\right), & \forall(x, t) \in \mathcal{D}, \\ u(x, 0)=u_{0}(x), & x \in=[0,1] \\ u(0, t)=s_{0}(t), & t \in(0, T], \\ u(1, t)=s_{1}(t), & t \in(0, T]\end{cases}
$$

where

$$
F\left(x, t, u, \frac{\partial u}{\partial x}\right)=(\beta+\gamma \beta) u^{2}-\beta u^{3}-\beta \gamma u-\alpha u \frac{\partial u}{\partial x} .
$$

Let us consider the homogenous part of Eq. (3.1) written as

$$
\left\{\begin{array}{l}
\frac{\partial u}{\partial t}-\varepsilon \frac{\partial^{2} u}{\partial x^{2}}=0, \quad \forall(x, t) \in \mathcal{D}, \\
u(x, 0)=u_{0}(x) \\
u(0, t)=s_{0}(t) \\
u(1, t)=s_{1}(t)
\end{array}\right.
$$


We look for a solution to the dimensionless heat equation of the form of Eq. (3.2), for $\varepsilon=1$, using the concept of separation of variables, we get the solution of Eq. (3.2) as

$$
u(x, t)=u_{0}(x) e^{-\pi^{2} t},
$$

which satisfies both the initial and boundary conditions.

To linearize Eq.(3.1), by applying the quasilinearization technique on the nonlinear term, for reasonable initial guess of the form of Eq. (3.3) is given by

$$
u^{(0)}(x, t)=u_{0}(x) e^{-\pi^{2} t} .
$$

Thus, the nonlinear term $F(x, t, u, \partial u / \partial x)$ can be linearized initially as;

$$
\begin{array}{r}
F\left(x, t, u^{(1)}, \frac{\partial u^{(1)}}{\partial x}\right) \cong F\left(x, t, u^{0}, \frac{\partial u^{0}}{\partial x}\right)+\left.\left(u^{(1)}-u^{(0)}\right) \frac{\partial F}{\partial u}\right|_{u^{0}} \\
+\left(\frac{\partial u^{(1)}}{\partial x}-\frac{\partial u^{(0)}}{\partial x}\right) \frac{\partial F}{\partial u^{\prime}} \mid \frac{\partial u^{0}}{\partial x}+\cdots
\end{array}
$$

Substituting Eq. (3.5) into Eq. (3.1) and inducing for iteration number $i=0,1, \ldots$, we obtain the linearized differential equation.

$$
\frac{\partial u^{(i+1)}}{\partial t}-\varepsilon \frac{\partial^{2} u^{(i+1)}}{\partial x^{2}}+a(x, t)^{(i)} \frac{\partial u^{(i+1)}}{\partial x}+b(x, t)^{(i)} u^{(i+1)}=f^{(i)}(x, t),
$$

where

$$
\begin{aligned}
& a^{(i)}(x, t)=\frac{\partial F}{\partial\left(\frac{\partial u}{\partial x}\right)} \mid\left(x, t, u^{(i)}, \frac{\partial u^{(i)}}{\partial x}\right)^{\prime} \\
& b^{(i)}(x, t)=-\frac{\partial F}{\partial u^{(i)}} \mid\left(x, t, u^{(i)}, \frac{\partial u^{(i)}}{\partial x}\right)^{\prime} \\
& f^{(i)}(x, t)=F\left(x, t, u^{(i)}, \frac{\partial u^{(i)}}{\partial x}\right)-u^{(i)} \frac{\partial F}{\partial u}\left|\left(x, t, u^{(i)}, \frac{\partial u^{(i)}}{\partial x}\right)-\frac{\partial u^{(i)}}{\partial x} \frac{\partial F}{\partial\left(\frac{\partial u}{\partial x}\right)}\right|\left(x, t, u^{(i)}, \frac{\partial u^{(i)}}{\partial x}\right)
\end{aligned}
$$

Let $M$ and $N$ be positive integers, then discretize the interval of $[0,1]$ and $[0, T]$ into $M$ and $N$ equal sub-intervals of the spatial and temporal direction respectively with uniform step length $h$ and $k$. Thus, the gird points $\left(x_{m}, t_{n}\right)$ can be generated by:

$$
\begin{cases}x_{m}=m h, \quad h=\frac{1}{M}, & m=0,1, \cdots, M, \\ t_{n}=n k, \quad k=\frac{T}{N}, & n=0,1, \cdots, N .\end{cases}
$$


At the fixed $(i+1)^{-t h}$ iteration, let us re-write equation (3.6) at the nodal point $\left(x_{m}, t_{n+\frac{1}{2}}\right)$ as:

$$
\left\{\begin{array}{l}
\mathcal{L}_{M}^{N} U \cong \frac{\partial U_{m}^{n+\frac{1}{2}}}{\partial t}-\varepsilon \frac{\partial^{2} U_{m}^{n+\frac{1}{2}}}{\partial x^{2}}+a_{m}^{n+\frac{1}{2}} \frac{\partial U_{m}^{n+\frac{1}{2}}}{\partial x}+b_{m}^{n+\frac{1}{2}} U_{m}^{n+\frac{1}{2}}=f_{m}^{n+\frac{1}{2}} \\
U(x, 0)=U_{0}\left(x_{m}\right), \quad x \in[0,1], \\
U\left(0, t_{n+1}\right)=s_{0}\left(t_{n+\frac{1}{2}}\right), \\
U\left(1, t_{n+1}\right)=s_{1}\left(t_{n+\frac{1}{2}}\right) .
\end{array}\right.
$$

Then, to get the finite difference approximation for $\frac{\partial u_{m}^{n+\frac{1}{2}}}{\partial t}$, we considered the Taylor's series expansion:

$$
\begin{aligned}
& U_{m}^{n+1}=u_{m}^{n+\frac{1}{2}}+\frac{k}{2} \frac{\partial u_{m}^{n+\frac{1}{2}}}{\partial t}+\frac{k^{2}}{8} \frac{\partial^{2} u_{m}^{n+\frac{1}{2}}}{\partial t^{2}}+\frac{k^{3}}{48} \frac{\partial^{3} u_{m}^{n+\frac{1}{2}}}{\partial t^{3}}+\ldots \\
& U_{m}^{n}=u_{m}^{n+\frac{1}{2}}-\frac{k}{2} \frac{\partial u_{m}^{n+\frac{1}{2}}}{\partial t}+\frac{k^{2}}{8} \frac{\partial^{2} u_{m}^{n+\frac{1}{2}}}{\partial t^{2}}-\frac{k^{3}}{48} \frac{\partial^{3} u_{m}^{n+\frac{1}{2}}}{\partial t^{3}}+\ldots
\end{aligned}
$$

Subtracting Eq. (3.10) from Eq. (3.9), gives the second-order finite difference approximation:

$$
\frac{\partial u_{m}^{n+\frac{1}{2}}}{\partial t}=\frac{U_{m}^{n+1}-U_{m}^{n}}{k}+\tau_{1}
$$

where the truncation term $\tau_{1}=-\frac{k^{2}}{24} \frac{\partial^{3} u_{m}^{n+\frac{1}{2}}}{\partial t^{3}}$.

Considering the remaining terms of Eq. (3.8), at the average of $n^{\text {th }}$ and $(n+1)^{\text {th }}$ time level, we have:

$$
\begin{aligned}
-\varepsilon \frac{\partial^{2} u_{m}^{n+\frac{1}{2}}}{\partial x^{2}}+a_{m}^{n+\frac{1}{2}} \frac{\partial U_{m}^{n+\frac{1}{2}}}{\partial x} & +b_{m}^{n+\frac{1}{2}} u_{m}^{n+\frac{1}{2}}-f_{m}^{n+\frac{1}{2}}=\frac{1}{2}\left(-\varepsilon \frac{U_{m+1}^{n}-2 U_{m}^{n}+U_{m-1}^{n}}{h^{2}}\right) \\
& +\frac{1}{2}\left(a_{m}^{n} \frac{U_{m+1}^{n}-U_{m-1}^{n}}{2 h}+b_{m}^{n} U_{m}^{n}-f_{m}^{n}-\varepsilon \frac{U_{m+1}^{n+1}-2 U_{m}^{n+1}+U_{m-1}^{n+1}}{h^{2}}\right) \\
& +\frac{1}{2}\left(a_{m}^{n+1} \frac{U_{m+1}^{n+1}-U_{m-1}^{n+1}}{2 h}+b_{m}^{n+1} U_{m}^{n+1}-f_{m}^{n+1}+\tau_{2}\right),
\end{aligned}
$$

where for the truncation term $\tau_{2}=-\frac{\varepsilon h^{2}}{12}\left(\frac{\partial^{4} u_{m}^{n}}{\partial x^{4}}+\frac{\partial^{4} u_{m}^{n+1}}{\partial x^{4}}\right)$.

Substituting Eqs. (3.11) and (3.12) into Eq. (3.8), and then re-written as the three-term recurrence relation in terms of the spatial direction and two-term recurrence relation in terms of the temporal direction as:

$$
L_{M}^{N} U_{m}^{n+1} \equiv E_{m}^{n+1} U_{m-1}^{n+1}-F_{m}^{n+1} U_{m}^{n+1}+G_{m}^{n+1} U_{m+1}^{n+1}=H_{m}^{n+1},
$$


where

$$
\begin{aligned}
& E_{m}^{n+1}=\frac{-\varepsilon}{h^{2}}-\frac{a_{m}^{n+1}}{2 h}, F_{m}^{n+1}=2 \frac{\varepsilon}{h^{2}}+\frac{2}{k}+b_{m}^{n+1}, G_{m}^{n+1}=\frac{-\varepsilon}{h^{2}}+\frac{a_{m}^{n+1}}{2 h}, \\
& H_{m}^{n+1}=f_{m}^{n+1}+f_{m}^{n}+\varepsilon \frac{U_{m+1}^{n}-2 U_{m}^{n}+U_{m-1}^{n}}{h^{2}}-a_{m}^{n+1} \frac{U_{m+1}^{n}-U_{m-1}^{n}}{2 h}-b_{m}^{n} U_{m}^{n}+\frac{2}{k} U_{m}^{n} .
\end{aligned}
$$

Note that, in this paper, we solve the problem under consideration numerically using Eq. (3.13) at the first iteration.

\section{Convergence analysis}

In this section, we carry out the stability analysis and derive the truncation error for the formulated scheme, and then we estimate its convergence.

Lemma 4.1. (Discrete Maximum Principle): Assume that the $i^{t h}$ iteration of the discrete function $\psi_{m}^{n}$ satisfies $\psi_{m}^{n} \geq 0$ on the discrete boundary $\Gamma_{M}^{N}$ and $\mathcal{L}_{M}^{N} \psi_{m}^{n} \geq 0, \forall\left(x_{m}, t_{n}\right) \in \mathcal{D}_{M}^{N}$. Then $\psi_{m}^{n} \geq$ $0, \forall\left(x_{m}, t_{n}\right) \in \overline{\mathcal{D}}_{M}^{N}$.

Proof. Suppose that there exists a point $\left(x_{m}^{\prime}, t_{n}^{\prime}\right) \in \mathcal{D}_{M}^{N}$ such that $\psi\left(x_{m}^{\prime}, t_{n}^{\prime}\right)=\min _{\overline{\mathcal{D}}_{M}^{N}} \psi<0$. Thus, from te given conditions on the boundary $\Gamma_{M}^{N}$, we have $\left(x_{m}^{\prime}, t_{n}^{\prime}\right) \notin \Gamma_{M}^{N}$, which implies that $\left(x_{m}^{\prime}, t_{n}^{\prime}\right) \in \mathcal{D}_{M}^{N}$. It follows from the definition of the point $\left(x_{m}^{\prime}, t_{n}^{\prime}\right) \in \overline{\mathcal{D}}_{M}^{N}$ that

$$
\frac{\partial \psi\left(x_{m}^{\prime}, t_{n}^{\prime}\right)}{\partial t^{\prime}}=0, \quad \frac{\partial \psi\left(x_{m}^{\prime}, t_{n}^{\prime}\right)}{\partial x^{\prime}}=0 \quad \text { and } \quad \frac{\partial^{2} \psi\left(x_{m}^{\prime}, t_{n}^{\prime}\right)}{\partial x^{\prime 2}} \geq 0 .
$$

Hence, $\mathcal{L}_{M}^{N} \psi_{m}^{n} \leq 0$. This is a contradiction to our assumption.

This lemma is used to prove the following uniform stability estimate.

Lemma 4.2. Uniform stability estimate for $E q$. (3.7) at any time level is estimated if $U_{m}^{n}$ is any function such that $U_{0}^{n}=U_{M}^{n}=0$, for $n=0,1,2, \cdots, N$. Then

$$
\left|U_{m}^{n}\right| \leq \frac{1}{\alpha} \max _{1 \leq m \leq M-1}\left|\mathcal{L}_{M}^{N} U_{m}^{n}\right|
$$

$a_{m}^{n} \geq \alpha \geq 0, \forall\left(x_{m}, t_{n}\right) \in \overline{\mathcal{D}}_{M}^{N}$.

Proof. Assume that $\psi^{ \pm}$be the barrier mesh function defined by:

$$
\left(\psi^{ \pm}\right)_{m}^{n}=\frac{1}{\alpha} \max _{1 \leq m \leq M-1}\left|\mathcal{L}_{\mathcal{E}, M}^{N} U_{m}^{n}\right| \pm U_{m}^{n}
$$

for $\left(\psi^{ \pm}\right)_{0}^{n}=\left(\psi^{ \pm}\right)_{M}^{n}=0$. Therefore, we have

$$
\mathcal{L}_{M}^{N}\left(\psi^{ \pm}\right)_{m}^{n}=\frac{a_{m}^{n}}{\alpha} \max _{1 \leq m \leq M-1}\left|\mathcal{L}_{M}^{N} U_{m}^{n}\right| \pm \mathcal{L}_{M}^{N} U_{m}^{n}
$$


Since $a_{m}^{n} \geq \alpha$ and from Lemma (4.1), we have $\mathcal{L}_{M}^{N} U_{m}^{n} \geq 0$, which concludes that $\mathcal{L}_{M}^{N}\left(\psi^{ \pm}\right)_{m}^{n} \geq$ 0 , on $\overline{\mathcal{D}}_{M}^{N}$. Hence, the required estimate.

The local truncation error $T(h, k)=\mathcal{L}\left(u_{m}^{n+1}-U_{m}^{n+1}\right)$ between the operator on the exact solution for (3.8) and approximate solution to Eq. (3.13) at the fixed $(i+1)-t h$ iteration is given by:

$$
\begin{aligned}
T(h, k)=\frac{\partial U_{m}^{n+\frac{1}{2}}}{\partial t} & -\varepsilon \frac{\partial^{2} U_{m}^{n+\frac{1}{2}}}{\partial x^{2}}+a_{m}^{n+\frac{1}{2}} \frac{\partial U_{m}^{n+\frac{1}{2}}}{\partial x}+b_{m}^{n+\frac{1}{2}} U_{m}^{n+\frac{1}{2}}-\frac{1}{2}\left(-\varepsilon \frac{U_{m+1}^{n}-2 U_{m}^{n}+U_{m-1}^{n}}{h^{2}}\right) \\
& -\frac{1}{2}\left(a_{m}^{n} \frac{U_{m+1}^{n}-U_{m-1}^{n}}{2 h}+b_{m}^{n} U_{m}^{n}-\varepsilon \frac{U_{m+1}^{n+1}-2 U_{m}^{n+1}+U_{m-1}^{n+1}}{h^{2}}\right) \\
& -\frac{1}{2}\left(a_{m}^{n+1} \frac{U_{m+1}^{n+1}-U_{m-1}^{n+1}}{2 h}+b_{m}^{n+1} U_{m}^{n+1}\right)-\frac{U_{m}^{n+1}-U_{m}^{n}}{k}
\end{aligned}
$$

By Taylor's series expansion, we have

$$
\begin{aligned}
& \left\{\begin{array}{l}
\frac{U_{m+1}^{n+1}-2 U_{m}^{n+1}+U_{m-1}^{n+1}}{h^{2}}=\frac{\partial^{2} U_{m}^{n+1}}{\partial x^{2}}+\frac{h^{2}}{12} \frac{\partial^{4} U_{m}^{n+1}}{\partial x^{4}}+\frac{h^{4}}{360} \frac{\partial^{6} U_{m}^{n+1}}{\partial x^{6}}+\ldots \\
\frac{U_{m+1}^{n}-2 U_{m}^{n}+U_{m-1}^{n}}{h^{2}}=\frac{\partial^{2} u_{m}^{n}}{\partial x^{2}}+\frac{h^{2}}{12} \frac{\partial^{4} U_{m}^{n}}{\partial x^{4}}+\frac{h^{4}}{360} \frac{\partial^{6} U_{m}^{n}}{\partial x^{6}}+\ldots
\end{array}\right. \\
& \left\{\begin{array}{l}
U_{m+1}^{n+1}-U_{m-1}^{n+1}=2 h \frac{\partial U_{m}^{n+1}}{\partial x}+O\left(h^{3}\right), \\
U_{m+1}^{n}-U_{m-1}^{n}=2 h \frac{\partial U_{m}^{n}}{\partial x}+O\left(h^{3}\right),
\end{array}\right. \\
& \frac{U_{m}^{n+1}-U_{m}^{n}}{k}=\frac{\partial U_{m}^{n+\frac{1}{2}}}{\partial t}+\frac{k^{2}}{24} \frac{\partial^{3} U_{m}^{n+\frac{1}{2}}}{\partial t^{3}}+\ldots
\end{aligned}
$$

Recall that the relations for the average is given by

$$
\left\{\begin{array}{l}
\frac{\partial^{2} U_{m}^{n+\frac{1}{2}}}{\partial x^{2}}=\frac{1}{2}\left(\frac{\partial^{2} U_{m}^{n+1}}{\partial x^{2}}+\frac{\partial^{2} U_{m}^{n}}{\partial x^{2}}\right) \\
a_{m}^{n+\frac{1}{2}} \frac{\partial U_{m}^{n+\frac{1}{2}}}{\partial x}=\frac{1}{2}\left(a_{m}^{n+1} \frac{\partial U_{m}^{n+1}}{\partial x}+a_{m}^{n} \frac{\partial U_{m}^{n}}{\partial x}\right) \\
b_{m}^{n+\frac{1}{2}} u_{m}^{n+\frac{1}{2}}=\frac{1}{2}\left(b_{m}^{n+1} u_{m}^{n+1}+b_{m}^{n} u_{m}^{n}\right)
\end{array}\right.
$$

Using Eqs. (4.2) - (4.5) into Eq. (4.1), we get

$$
T(h, k)=\frac{\varepsilon h^{2}}{24}\left(\frac{\partial^{4} U_{m}^{n+1}}{\partial x^{4}}+\frac{\partial^{4} U_{m}^{n}}{\partial x^{4}}\right)+\frac{k^{2}}{24} \frac{\partial^{3} U_{m}^{n+\frac{1}{2}}}{\partial t^{3}}+\ldots
$$


Hence, from Eq. (4.6), we have

$$
|T(h, k)|_{\infty} \leq C_{1} h^{2}+C_{2} k^{2} \leq C\left(h^{2}+k^{2}\right)
$$

where

$$
C_{1}=\left|\frac{\varepsilon}{24}\left(\frac{\partial^{4} U_{m}^{n+1}}{\partial x^{4}}+\frac{\partial^{4} U_{m}^{n}}{\partial x^{4}}\right)\right|_{\infty} \quad \text { and } \quad C_{2}=\left|\frac{1}{24} \frac{\partial^{3} U_{m}^{n+\frac{1}{2}}}{\partial t^{3}}\right|_{\infty} .
$$

Thus, the constant $C$ is independent of the mesh parameters $h$ and $k$.

\section{Numerical illlustrations and discussions}

In this section, we considered model examples of singularly perturbed Burger-Huxley equations to validate our theoretical results. These examples have been chosen since they have widely discussed in the litrature under consideration. The exact solution for the considered examples are not available. Hence, the maximum absolute errors are calculated by double mesh principle, $[1,28,29]$, given by:

$$
E_{M}^{N}=\max _{\forall\left(x_{m}, t_{n}\right) \in \overline{\mathcal{D}}}\left|U_{m}^{n}-U_{2 m}^{2 n}\right|,
$$

where $U_{m}^{n}$ and $U_{2 m}^{2 n}$ are approximate solutions evaluated on $\overline{\mathcal{D}}_{M}^{N}$ and $\overline{\mathcal{D}}_{2 M}^{2 N}$ respectively. The corresponding rate of convergences are determined by:

$$
R_{M}^{N}=\frac{\log \left(E_{M}^{N}\right)-\log \left(E_{2 M}^{2 N}\right)}{\log (2)} .
$$

Example 5.1. Consider the following singularly perturbed Burgers-Huxley equation

$$
\begin{cases}\frac{\partial u}{\partial t}+u \frac{\partial u}{\partial x}-\varepsilon \frac{\partial^{2} u}{\partial x^{2}}-(1-u)(u-0.5) u=0, & \forall(x, t) \in(0,1) \times(0,1], \\ u(x, 0)=x\left(1-x^{2}\right), & 0<x<1, \\ u(0, t)=u(1, t)=0, & t \in[0,1] .\end{cases}
$$

Example 5.2. Consider the following singularly perturbed Burgers' equation

$$
\begin{cases}\frac{\partial u}{\partial t}+u \frac{\partial u}{\partial x}-\varepsilon \frac{\partial^{2} u}{\partial x^{2}}=0, & \forall(x, t) \in(0,1) \times(0,1], \\ u(x, 0)=x\left(1-x^{2}\right), & 0<x<1, \\ u(0, t)=u(1, t)=0, & t \in[0,1] .\end{cases}
$$

The numerical results are presented in Tables 1-4, and Figures 1 and 2.

Tables 1 and 3 shows the maximum absolute errors that demonstrates the validity of the present method, and errors are monotonically decreasing (see Figure 3) behavior 

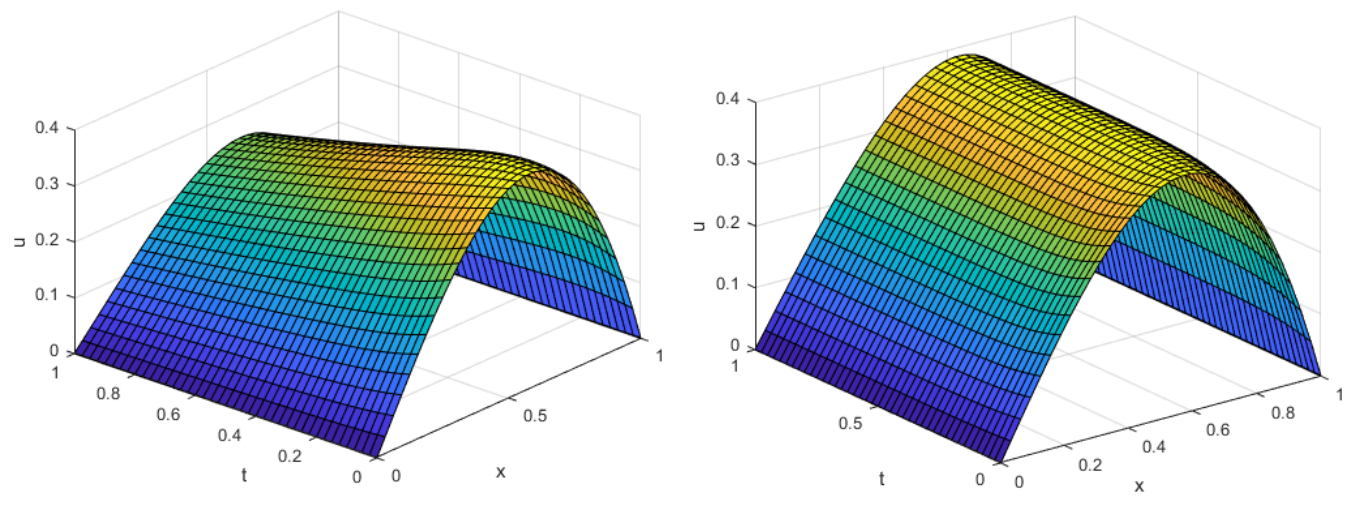

Figure 1: Solution profiles for Examples 5.1 and 5.2 respectively, when $M=64, N=40, \varepsilon=2^{-18}$.
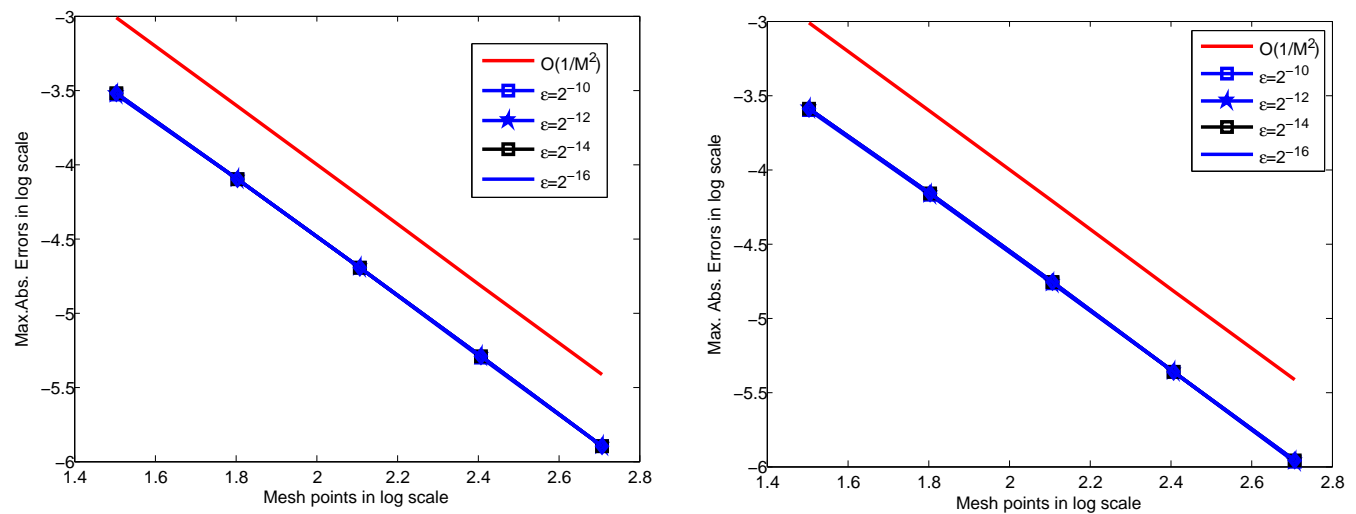

Figure 2: Log-log plots for Example 5.1 and 5.2 respectively.
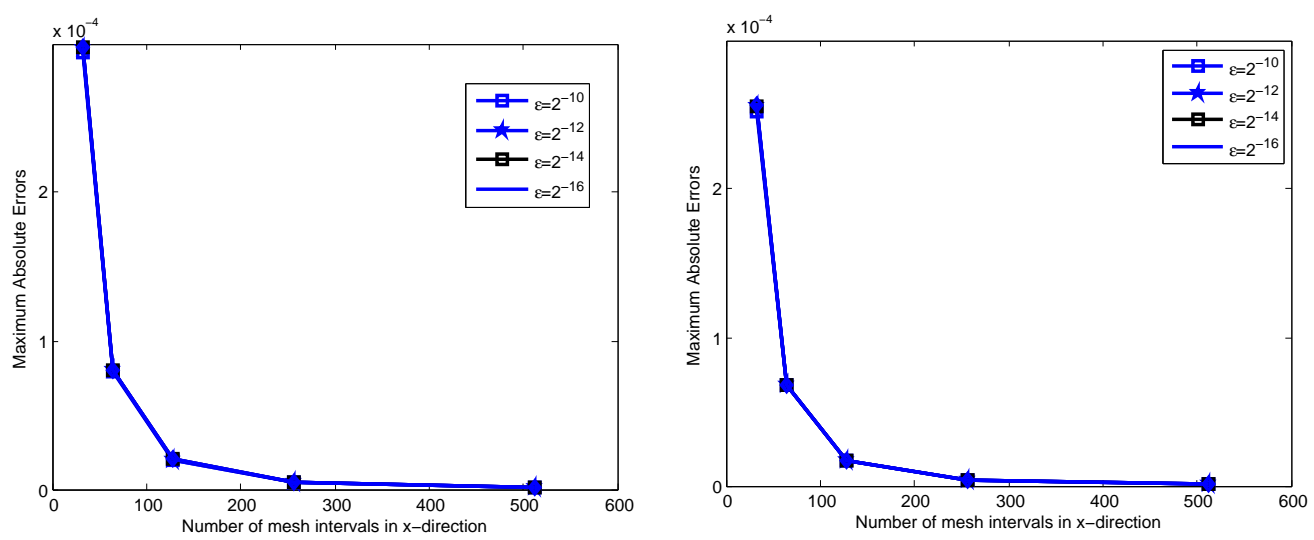

Figure 3: Maximum absolute errors via number of mesh points for Example 5.1 and 5.2 respectively. 
Table 1: Comparison of maximum absolute errors for Example 5.1.

\begin{tabular}{|l|ccccc|}
\hline$\downarrow \varepsilon M / N \rightarrow$ & $32 / 20$ & $64 / 40$ & $128 / 80$ & $256 / 160$ & $512 / 320$ \\
\hline Present Method & & & & & \\
$2^{-10}$ & $2.9393 e-04$ & $7.9356 e-05$ & $2.0013 e-05$ & $5.0159 e-06$ & $1.2556 e-06$ \\
$2^{-12}$ & $2.9695 e-04$ & $7.9882 e-05$ & $2.0146 e-05$ & $5.0535 e-06$ & $1.2646 e-06$ \\
$2^{-14}$ & $2.9770 e-04$ & $8.0013 e-05$ & $2.0180 e-05$ & $5.0629 e-06$ & $1.2668 e-06$ \\
$2^{-16}$ & $2.9789 e-04$ & $8.0046 e-05$ & $2.0188 e-05$ & $5.0653 e-06$ & $1.2674 e-06$ \\
$2^{-18}$ & $2.9794 e-04$ & $8.0054 e-05$ & $2.0190 e-05$ & $5.0659 e-06$ & $1.2675 e-06$ \\
Results in [1] & & & & & \\
$2^{-10}$ & $9.3183 e-02$ & $7.0120 e-02$ & $4.4773 e-02$ & $2.0546 e-02$ & $1.0545 e-02$ \\
$2^{-12}$ & $1.7017 e-01$ & $1.0083 e-01$ & $6.2216 e-02$ & $3.9526 e-02$ & $2.0493 e-02$ \\
$2^{-14}$ & $2.0410 e-01$ & $1.6703 e-01$ & $9.2110 e-02$ & $5.2580 e-02$ & $2.8766 e-02$ \\
$2^{-16}$ & $2.0450 e-01$ & $1.5975 e-01$ & $1.2612 e-01$ & $6.9772 e-02$ & $3.8531 e-02$ \\
$2^{-18}$ & $2.5614 e-01$ & $2.1031 e-01$ & $1.3406 e-01$ & $8.5618 e-02$ & $4.8834 e-02$ \\
\hline
\end{tabular}

Table 2: Comparison of rate of convergence for Example 5.1.

\begin{tabular}{|l|cccc|}
\hline$\downarrow \varepsilon M / N \rightarrow$ & $32 / 20$ & $64 / 40$ & $128 / 80$ & $256 / 160$ \\
\hline $2^{-10}$ & 1.8891 & 1.9874 & 1.9964 & 1.9981 \\
$2^{-12}$ & 1.8943 & 1.9874 & 1.9951 & 1.9986 \\
$2^{-14}$ & 1.8956 & 1.9873 & 1.9949 & 1.9988 \\
$2^{-16}$ & 1.8959 & 1.9873 & 1.9948 & 1.9988 \\
$2^{-18}$ & 1.8960 & 1.9873 & 1.9948 & 1.9988 \\
Results in [1] & & & & \\
$2^{-10}$ & 0.4102 & 0.6472 & 1.1238 & 0.9623 \\
$2^{-12}$ & 0.7550 & 0.6966 & 0.6544 & 0.9477 \\
$2^{-14}$ & 0.2892 & 0.8587 & 0.8088 & 0.8701 \\
$2^{-16}$ & 0.3563 & 0.3410 & 0.8540 & 0.8566 \\
$2^{-18}$ & 0.2844 & 0.6496 & 0.6469 & 0.8100 \\
\hline
\end{tabular}

with increasing the number of intervals which confirm the convergence of the method. Further, Tables 2 and 4 validates that the the rate of convergence described in Eq. (4.7). Thus, the proposed method with higher-order convergent than some existing methods in the literature.

Furthermore, the method gives more accurate solution than some existing methods in the literature. Figure 1 verifies that the physical behavior of the solutions for both Examples. Log-log plot presented in Figure 2 for the modeled examples under consideration.

\section{Conclusion}

In this paper, higher-order implicit finite difference scheme is presented for solving the singularly perturbed Burger-Huxley equation. At first, the nonlinear terms are linearized 
Table 3: Comparison of maximum absolute errors for Example 5.2.

\begin{tabular}{|l|ccccc|}
\hline$\downarrow \varepsilon M / N \rightarrow$ & $32 / 20$ & $64 / 40$ & $128 / 80$ & $256 / 160$ & $512 / 320$ \\
\hline $2^{-10}$ & $2.5222 e-04$ & $6.7547 e-05$ & $1.7077 e-05$ & $4.2892 e-06$ & $1.0730 e-06$ \\
$2^{-12}$ & $2.5527 e-04$ & $6.8045 e-05$ & $1.7231 e-05$ & $4.3246 e-06$ & $1.0818 e-06$ \\
$2^{-14}$ & $2.5603 e-04$ & $6.8169 e-05$ & $1.7270 e-05$ & $4.3334 e-06$ & $1.0842 e-06$ \\
$2^{-16}$ & $2.5622 e-04$ & $6.8201 e-05$ & $1.7280 e-05$ & $4.3356 e-06$ & $1.0847 e-06$ \\
$2^{-18}$ & $2.5627 e-04$ & $6.8209 e-05$ & $1.7282 e-05$ & $4.3362 e-06$ & $1.0849 e-06$ \\
Results in [1] & & & & & \\
$2^{-10}$ & $8.3339 e-02$ & $6.6120 e-02$ & $4.0769 e-02$ & $1.9284 e-02$ & $9.1775 e-03$ \\
$2^{-12}$ & $1.8762 e-01$ & $8.4106 e-02$ & $5.7234 e-02$ & $3.8309 e-02$ & $1.9041 e-02$ \\
$2^{-14}$ & $1.9755 e-01$ & $1.5347 e-01$ & $8.3864 e-02$ & $4.6945 e-02$ & $2.5508 e-02$ \\
$2^{-16}$ & $2.1340 e-01$ & $1.5016 e-01$ & $1.1582 e-01$ & $6.1958 e-02$ & $3.3441 e-02$ \\
$2^{-18}$ & $2.8299 e-01$ & $1.7036 e-01$ & $1.1805 e-01$ & $7.4251 e-02$ & $4.1879 e-02$ \\
\hline
\end{tabular}

Table 4: Comparison of rate of convergence for Example 5.2.

\begin{tabular}{|l|cccc|}
\hline$\downarrow \mathcal{E} M / N \rightarrow$ & $32 / 20$ & $64 / 40$ & $128 / 80$ & $256 / 160$ \\
\hline $2^{-10}$ & 1.9007 & 1.9838 & 1.9933 & 1.9991 \\
$2^{-12}$ & 1.9075 & 1.9815 & 1.9944 & 1.9991 \\
$2^{-14}$ & 1.9091 & 1.9808 & 1.9947 & 1.9989 \\
$2^{-16}$ & 1.9095 & 1.9807 & 1.9948 & 1.9989 \\
$2^{-18}$ & 1.9096 & 1.9807 & 1.9948 & 1.9989 \\
Results in [1] & & & & \\
$2^{-10}$ & 0.3339 & 0.6976 & 1.0801 & 1.0712 \\
$2^{-12}$ & 1.1575 & 0.5553 & 0.6544 & 1.0086 \\
$2^{-14}$ & 0.3643 & 0.8718 & 0.8371 & 0.8800 \\
$2^{-16}$ & 0.5076 & 0.3746 & 0.9025 & 0.8897 \\
$2^{-18}$ & 0.7322 & 0.5292 & 0.6689 & 0.8262 \\
\hline
\end{tabular}

by quasilinearization technique. Then, we use average finite difference approximation for the discretization of derivatives. A second-order point-wise convergent error estimations are established. It is provided that from numerical results, the proposed method gives a better accurate solution with higher order of convergence than some existing methods in the literature. Therefore, the proposed method is an implicit finite difference scheme of second-order converegnt and gives accurate solution for solving the singularly perturbed Burger-Huxley equation.

\section{Acknowledgement}

We thank Jimma University for the necessary support, reviwers and editors of the Journals of Partial Differential Equations. 


\section{References}

[1] Liu L. B., Liang Y., Zhang J. and Bao X., A robust adaptive grid method for singularly perturbed Burger-Huxley equations. Electronic Research Archive, 28 (4) (2020), 1439-1457.

[2] Duan L., Lu Q., Bursting oscillations near codimension-two bifurcations in the Chay Neuron model. International Journal of Nonlinear Sciences and Numerical Simulation, 7 (1) (2006), 59-64.

[3] Liu S., Fan T. and Lu Q., The spike order of the winnerless competition (WLC) model and its application to the inhibition neural system. International Journal of Nonlinear Sciences and Numerical Simulation, 6 (2) (2005), 133-138.

[4] Aronson D. G., Weinberger H. F., Multidimensional nonlinear diffusion arising in population genetics. Advances in Mathematics, 30 (1) (1978), 33-76.

[5] Zhang G. J., Xu J. X., Yao H. and Wei R. X., Mechanism of bifurcation-dependent coherence resonance of an excitable neuron model. International Journal of Nonlinear Sciences and Numerical Simulation, 7 (4) (2006), 447-450.

[6] Satsuma J., Exact Solutions of Burgers' Equation with Reaction Terms. Topics in Soliton Theory and Exact Solvable Nonlinear Equations, 1987.

[7] Wang X. Y., Zhu Z. S. and Lu Y. K., Solitary wave solutions of the generalised BurgersHuxley equation. Journal of Physics A: Mathematical and General, 23 (3) (1990), 271-274.

[8] Wazwaz A. M., Travelling wave solutions of generalized forms of Burgers, Burgers-KdV and Burgers-Huxley equations. Applied Mathematics and Computation, 169 (1) (2005), 639-656.

[9] Bullo T. A., Duressa G. F. and Degla G., Accelerated fitted operator finite difference method for singularly perturbed parabolic reaction-diffusion problems. Computational Methods for Differential Equations, 9 (3) (2021), 886-898.

[10] Bullo T. A., Duressa G. F. and Degla G. A., Robust finite difference method for singularly perturbed two-parameter parabolic convection-diffusion problems. International Journal of Computational Methods, 18 (2) (2021) 2050034 (17 pages).

[11] Kabeto M. J., Duressa G. F., Robust numerical method for singularly perturbed semilinear parabolic differential difference equations. Mathematics and Computers in Simulation, 188 (2021), 537-547.

[12] Bullo T. A., Degla G. A. and Duressa G. F., Uniformly convergent higher-order finite difference scheme for singularly perturbed parabolic problems with non-smooth data. Journal of Applied Mathematics and Computational Mechanics, 20 (1) (2021), 5-16.

[13] Woldaregay M. M., Duressa G. F., Uniformly convergent numerical method for singularly perturbed delay parabolic differential equations arising in computational neuroscience. Kragujevac Journal of Mathematics, 46 (1) (2022), 65-84.

[14] Hashim I., Noorani M. S. M. and Batiha B., A note on the Adomian decomposition method for the generalized Huxley equation. Applied Mathematics and Computation, 181 (2) (2006), 1439-1445.

[15] Hashim I., Noorani M. S. M. and Al-Hadidi M. S., Solving the generalized Burgers-Huxley equation using the Adomian decomposition method. Mathematical and Computer Modelling, 43 (11-12) (2006), 1404-1411.

[16] Ismail H. N., Raslan K. and Abd Rabboh A. A., Adomian decomposition method for Burger's-Huxley and Burger's-Fisher equations. Applied Mathematics and Computation, 159 (1) (2004), 291-301.

[17] Javidi M., Golbabai A., A new domain decomposition algorithm for generalized Burger'sHuxley equation based on Chebyshev polynomials and preconditioning. Chaos, Solitons and 
Fractals, 39(2) (2009), 849-857.

[18] Batiha B., Noorani M. S. M. and Hashim I., Numerical simulation of the generalized Huxley equation by He's variational iteration method. Applied Mathematics and Computation, 186 (2) (2007), 1322-1325.

[19] Mohanty R. K., Dai W. and Liu D., Operator compact method of accuracy two in time and four in space for the solution of time dependent Burgers-Huxley equation. Numerical Algorithms, 70 (3) (2015), 591-605.

[20] Sari M., Grarslan G. and Zeytinoglu A., High-order finite difference schemes for numerical solutions of the generalized Burgers-Huxley equation. Numerical Methods for Partial Differential Equations, 27 (5) (2011), 1313-1326.

[21] Darvishi M. T., Kheybari S. and Khani F., Spectral collocation method and Darvishi's preconditionings to solve the generalized Burgers-Huxley equation. Communications in Nonlinear Science and Numerical Simulation, 13 (10) (2008), 2091-2103.

[22] Javidi M., A numerical solution of the generalized Burger's-Huxley equation by spectral collocation method. Applied mathematics and computation, 178(2) (2006), 338-344.

[23] Mohammadi R., B-spline collocation algorithm for numerical solution of the generalized Burger's-Huxley equation. Numerical Methods for Partial Differential Equations, 29 (4) (2013), 1173-1191.

[24] Mittal R. C., Tripathi A., Numerical solutions of generalized Burgers-Fisher and generalized Burgers-Huxley equations using collocation of cubic B-splines. International Journal of Computer Mathematics, 92 (5) (2015), 1053-1077.

[25] Khattak A. J., A computational meshless method for the generalized Burger's-Huxley equation. Applied Mathematical Modelling, 33 (9) (2009), 3718-3729.

[26] Kaushik A., Sharma M. D., A uniformly convergent numerical method on non-uniform mesh for singularly perturbed unsteady Burger-Huxley equation. Applied mathematics and computation, 195 (2) (2008), 688-706.

[27] Gupta V., Kadalbajoo M. K., A singular perturbation approach to solve Burgers-Huxley equation via monotone finite difference scheme on layer-adaptive mesh. Communications in Nonlinear Science and Numerical Simulation, 16 (4) (2011), 1825-1844.

[28] Turuna D. A., Woldaregay M. M. and Duressa G. F., Uniformly convergent numerical method for singularly perturbed convection-diffusion problems. Kyungpook Mathematical Journal, 60 (3) (2020), 629-645.

[29] Bullo T., Duressa G. and Degla G., Higher order fitted operator finite difference method for two-parameter parabolic convection-diffusion problems. International Journal of Engineering and Applied Sciences, 11 (4) (2019), 455-467. 